\title{
PHF5A represents a bridge protein between splicing proteins and ATP-dependent helicases and is differentially expressed during mouse spermatogenesis
}

\author{
T. Rzymski $^{\mathrm{a}} \quad$ P. Grzmil ${ }^{\mathrm{a}} \quad$ A. Meinhardt ${ }^{\mathrm{b}} \quad$ S. Wolf ${ }^{\mathrm{a}} \quad$ P. Burfeind ${ }^{\mathrm{a}}$ \\ ${ }^{a}$ Institute of Human Genetics, University of Goettingen, Goettingen, \\ ${ }^{b}$ Department of Anatomy and Cell Biology, Justus-Liebig-University Giessen, Giessen (Germany)
}

Accepted in revised form for publication by M. Schmid, 9 April 2008.

\begin{abstract}
PHF5A is a highly conserved protein from yeast to man, and based on studies in yeast, it was suggested that the homologous protein RDS3P in S. cerevisiae takes part in the organization of U2 snRNP particles. By using the yeast two-hybrid assay we could demonstrate that PHF5A interacted both with ATP-dependent helicases EP400 and DDX1 and with arginine-serine (RS)-rich domains of splicing factors U2AF1 and SFRS5 in mouse. Furthermore, domain interaction studies revealed that PHF5A interaction with EP400 and DDX1 is restricted to the $\mathrm{N}$-terminal part of PHF5A, whereas the C-terminal region of PHF5A was found to be responsible for the association with U2AF1 and SFRS5. By using the yeast three-hybrid assay, we could further show that both EP400 and DDX1 interacted only indi-
\end{abstract}

PHF5A resembles a $12.4 \mathrm{kDa}$ nuclear protein, which is highly conserved among eukaryotes, sharing over $70 \%$ identity with proteins from a wide variety of organisms from yeast to man. In a recent study it was shown that $P h f 5 a$ is expressed ubiquitously in prenatal and postnatal murine tissues and that the mouse PHF5A protein is localized in the

\footnotetext{
This work was supported by a grant (SFB 271) from the Deutsche Forschungsgemeinschaft.

T.R. and P.G. contributed equally to this work.

Request reprints from Dr. Peter Burfeind

Institute of Human Genetics, University of Goettingen

Heinrich Dueker-Weg 12, DE-37073 Goettingen (Germany)

telephone: +49551 397595; fax: +49551399303

e-mail: pburfei@gwdg.de
}

rectly with U2AF1 and SFRS5 proteins via the bridge protein PHF5A. The subcellular localization of a PHF5A-GFP fusion protein was predominantly observed in the nucleus and, in addition, PHF5A co-localized with both U2AF1 and SFRS5 proteins in nuclear speckles of NIH3T3 cells. Moreover, expression analyses demonstrated that PHF5A and U2AF1 gene expression coincided in spermatocytes during murine spermatogenesis and interaction between these proteins was also detectable in the spermatocyte-specific cell line GC-4spc by using in vivo co-immunoprecipitation studies. Taken together, our results indicate that PHF5A resembles a protein which interacts with splicing factors U2AF1 and SFRS5 and helicases EP400 and DDX1 and functions as a bridge protein between these proteins.

Copyright $\odot 2008$ S. Karger AG, Basel nucleus in a non-homogenous pattern. Therefore, it was proposed that the PHF5A protein could play a role in both basic and essential cellular functions, possibly acting as a chromatin-associated protein (Trappe et al., 2002a). Furthermore, the homologous protein PHF5A in rat has been demonstrated to bind to the promoter of the connexin 43 gene, thereby increasing its expression in response to estrogen induction (Oltra et al., 2003). In addition, evidences were presented that PHF5A was essential for morphogenetic development in C. elegans and that the Phf5a gene exhibited a tissue- and stage-specific pattern of expression (Trappe et al., 2002b). Therefore, it was suggested that PHF5A could play a very complex role as a general transcriptional activator of different genes.

In eukaryotic systems transcripts are usually subjected to intensive processing including capping, splicing, poly- 
adenylation, and nuclear export prior to translation. PremRNA processing is highly coordinated with gene expression, and proteins involved in the splicing process are often localized in conjunction with the polymerase II holoenzyme complex (Wilson et al., 1996; Neish et al., 1998). In RNA splicing noncoding sequences are removed which typically requires a large ribonucleoprotein complex called the spliceosome and multiple auxiliary factors. Spliceosomes are very dynamic and complex structures and, among all of the proteins involved in splicing, proteins containing an arginine-serine-rich domain (RS domain) are one of their most characteristic and prominent proteins. RS domains are typically localized in the C-terminal part of serine/arginine-rich (SR) proteins and other related proteins, e.g. the U2AF-heterodimer which is composed of a $35 \mathrm{kDa}$ subunit (namely $\mathrm{U} 2 \mathrm{AF} 1$ or $\mathrm{U}_{2} \mathrm{AF}^{35}$ ) and a $65 \mathrm{kDa}$ subunit (namely U2AF2 or U2AF ${ }^{65}$ ) (Ruskin et al., 1988). The superfamily of ATP-dependent helicases is another rapidly expanding group of proteins involved in RNA metabolism (reviewed in Luking et al., 1998; Tanner and Linder, 2001). Whereas some of these proteins are exclusively involved in RNA metabolism, others have been shown to act more universal and are capable of utilizing RNA-DNA or DNA substrates as well. The DEAD box, which is characterized by the conserved amino acid motif Asp-Glu-Ala-Asp, is a common component of RNA helicases unwinding RNA duplexes in an ATPdependent manner. This vast family of proteins is involved in each step of RNA metabolism and processing including transcription, pre-mRNA splicing, spliceosome assembly, transport to the cytoplasm, translation, RNA decay, and gene expression in cell organelles. The DDX1 protein is a member of the DEAD box family of putative RNA helicases (Godbout and Squire, 1993) and this protein has been found to co-localize with the cleavage bodies, nuclear structures enriched in proteins involved in $3^{\prime}$ processing of mRNA (Bleoo et al., 2001). The EP400 (also known as mDomino) protein is a SWI2/SNF2 type ATP helicase family member assigned within the large superfamily of DEXD/H-ATPases and has been implicated in ATP-dependent remodeling of chromatin (Ogawa et al., 2003).

The relations between different components of the RNA processing machinery are a key to understand both the constitutive and the alternative splicing process and its coordination with gene expression. In this report we present the first evidence that murine PHF5A interacts with two ATPdependent helicases, namely DDX1 and EP400. In addition, we could show that the N-terminal fragment of PHF5A associates with two RS domains of the U2 snRNP proteins U2AF1 and SFRS5. Furthermore, yeast three-hybrid experiments demonstrate that PHF5A acts as a bridge protein between SR proteins and ATP-dependent helicases. Interaction of PHF5A with U2AF1 is stable in a wide broad of salt concentration and was detectable in both in vitro and in vivo systems. Moreover, PHF5A could be partially co-localized in nuclear speckles with both U2AF1 and SFRS5. During spermatogenesis PHF5A was found to be mainly present in primary spermatocytes indicating an important role of PHF5A in meiosis.

\section{Materials and methods}

Yeast two-hybrid and yeast three-hybrid assay

The yeast two-hybrid experiment was performed using the Matchmaker System 3 (BD Biosciences Clontech). Yeast cultures and two-hybrid procedures were carried out according to standard methods. The bait plasmid was introduced into the AH 109 yeast strain, and transformants were selected on SD plates lacking tryptophan (-Trp). To screen for interaction partners, the pre-transformed murine 11 day-old embryo Matchmaker library (BD Biosciences Clontech) was mixed with the yeast strain containing the bait (Phf5a) construct. Mating of both strains occurred during a $24 \mathrm{~h}$ incubation step at $30^{\circ} \mathrm{C}$ in $2 \times$ YPDA medium. Mating cultures were plated on SD minimal medium lacking leucine, tryptophan, histidine, adenine (-Leu, -Try, -His, -Ade) and supplemented with X-gal. After four days blue colonies were selected and library inserts were amplified by colony PCR and sequenced from both ends. The identity of individual clones was determined using the BLAST program (http://www.ncbi.nlm.nih.gov/ BLAST/) to screen the GenBank database (NCBI). Interactions of RS domain proteins with PHF5A and domain mapping experiments were investigated by co-transformation of appropriate $\mathrm{BD}$ (binding domain) and $\mathrm{AD}$ (activation domain) constructs into the yeast strain AH109. Selection of transformants was performed as described above. In the yeast three-hybrid assay cells were transformed with the bicistronic construct pBridge (BD Biosciences Clontech) and pGADT7. Selection of positive clones was performed as described above. Expression of the bridge protein was repressed by the addition of $1 \mathrm{mM}$ methionine to the selective culture plate.

\section{$\alpha$-Galactosidase ( $\alpha$-Gal) quantitative assay}

Yeast cells were cultured in selective SD medium (-His, -Try, -Leu) at $30^{\circ} \mathrm{C}$ for $16-18 \mathrm{~h}$ with shaking. After the incubation step, the optical density (OD) at $600 \mathrm{~nm}$ was measured and $1.0 \mathrm{ml}$ of the culture was centrifuged to pellet the cells completely. The supernatant was transferred and $48 \mu \mathrm{l}$ assay buffer $(0.5 \mathrm{M} \mathrm{NaOAc} \mathrm{pH} 4.5,100 \mathrm{mM}$ PNP $\alpha$-Gal, 2:1 (v/v) ratio) was added to $16 \mu$ l of the sample. The reaction was stopped after $30 \mathrm{~min}$ by adding $136 \mu \mathrm{l}$ of $1 \mathrm{M} \mathrm{Na}_{2} \mathrm{CO}_{3}$. Each experiment was performed three times in triplicate. The OD was recorded at $410 \mathrm{~nm}$ and the amount of $\alpha$-galactosidase was calculated.

\section{Immunoprecipitation}

The in vitro immunoprecipitation assay was used to confirm protein interactions. HA and c-Myc tagged fusion proteins were labeled with ${ }^{35} \mathrm{~S}$-methionine and expressed using the TNT ${ }^{\circledR}$ T7 Coupled Reticulocyte Lysate System (Promega), and pGADT7 and pGBKT7 plasmids were used as a template. Subsequently, $10 \mu \mathrm{l}$ of in vitro translated ${ }^{35} \mathrm{~S}$-labeled proteins were combined in one tube and incubated for $1 \mathrm{~h}$ at room temperature. Next, $10 \mu \mathrm{l}$ of c-Myc monoclonal antibody or HA polyclonal antibody (BD Biosciences Clontech) were added and incubated for $1 \mathrm{~h}$ at room temperature. Meanwhile, protein A beads (BD Biosciences Clontech) were washed in PBS. Beads and reaction mixtures were incubated with constant rotation for an additional hour. Unbound proteins were removed by five times washing in buffer 1 (150 $\mathrm{mM} \mathrm{NaCl}, 50 \mathrm{mM}$ Tris, $\mathrm{pH}$ 7.4, 2 mM EDTA, pH 8.0, 1\% Triton X$100 / 10 \%$ glycerol/2 $\mathrm{mM} \mathrm{DTT}$ ) and three times washing in buffer 2 (150 mM NaCl/50 mM Tris, pH 7.4). Finally, the beads were resuspended in $20 \mu \mathrm{l}$ SDS PAGE-loading buffer and proteins were denatured by heating at $80^{\circ} \mathrm{C}$ and loaded onto a SDS-PAGE minigel. After electrophoresis the gel was fixed and rinsed with the Amersham Amplify Fluorographic Reagent (GE Healthcare Life Science) according to the manufacturer's instruction, dried under vacuum and exposed to an X-ray film.

\section{In vivo coimmunoprecipitation}

Immortalized spermatocyte-specific GC-4spc cells were cultured as we described previously (Tascou et al., 2000). GC-4spc cells were washed, harvested and lysed for $30 \mathrm{~min}$ in lysis buffer $(50 \mathrm{mM}$ Tris $\mathrm{pH}$ 7.4, $150 \mathrm{mM} \mathrm{NaCl}, 2 \mathrm{mM}$ EDTA, 0.1\% NP40, 2.5\% glycerol, 2 mM DTT, $25 \mu \mathrm{g} / \mathrm{ml}$ aprotinin, $10 \mu \mathrm{g} / \mathrm{ml}$ pepstatin, $10 \mu \mathrm{g} / \mathrm{ml}$ chymostatin, $10 \mu \mathrm{g} /$ $\mathrm{ml}$ leupeptin, $1 \mathrm{mM}$ PMSF). Lysates were incubated overnight at $4^{\circ} \mathrm{C}$ 
with anti-PHF5A antibodies and protein A agarose beads (BD Biosciences Clontech). Unbound material was washed from the beads by rocking five times $10 \mathrm{~min}$ with lysis buffer. Bound proteins were separated on $4-12 \%$ SDS polyacrylamide gels and detected by Western blotting.

\section{Generation of constructs}

The BD/PHFa construct used for screening the yeast two-hybrid library was generated by fusing the complete open reading frame (ORF) of Phf5a (Accession No.: NM_026737) in frame to the pGBKT7 vector (BD Biosciences Clontech).

Constructs AD/Sfrs5-RS, AD/U2af1, AD/Ep400-RS, AD/Ddx1-RS were prepared by digestion with $\mathrm{NcoI} / \mathrm{S} f \mathrm{iI}$ of colony PCR products obtained from yeast colonies no. 94 (containing a cDNA fragment of $S$ frs 5 (Accession No.:NM_009159) from nucleotide position 659-832), no. 35 (containing a cDNA fragment of U2af1 (Accession No.:NM_024187) from nucleotide position 21-740), no. 100 (containing a cDNA fragment of Ep400 (Accession No.: AB092695) from nucleotide position 1880-2516), no. 81 (containing a cDNA fragment of $D d x 1$ (Accession No.:NM_134040) from nucleotide position 1660-2303), respectively, and cloned in frame into the pGADT7 vector (BD Biosciences Clontech).

Constructs containing RS domains of Sfrs1 (also known as ASF/ SF2, Accession No.: NM_173374; cDNA fragment from nucleotide position 697-882), Sfrs3 (also known as SRp20, Accession No.: NM_ 013663; cDNA fragment from nucleotide position 355-612), Sfrs9 (also known as SRp30c, Accession No.:NM_025573; cDNA fragment from nucleotide position 406-801) and U2aff (cDNA fragment from nucleotide position 540-740) were generated by RT-PCR, and cloned in frame into the pGADT7 vector.

Plasmids coding different peptide segments of $P h f 5 a, \mathrm{Phfa} \Delta 81-110$ (from nucleotide position 40-284), Phf5a $\Delta 1-28,81-110$ (from nucleotide position 129-284), Phf5a $\Delta$ 1-28 (from nucleotide position 129 371), Phf5a $\Delta 38-110$ (from nucleotide position $40-149$ ), Phf5a $\Delta 58-110$ (from nucleotide position 40-215), Phf5a $\Delta 1-50$ (from nucleotide position 195-371) and Phf5a $\Delta 1-80$ (from nucleotide position 285-371) were generated by PCR and cloned into the pGBKT7 vector and the pET41 vector (Novagen), respectively.

Construct DsRed/Sfrs5 was obtained by RT-PCR and cloning of the fragment corresponding to the ORF of the Sfrs 5 in frame into the pDsRed1-C1 vector (BD Biosciences Clontech). The pEGFP/Phf5a construct has been previously described (Trappe et al., 2002a).

Splicing variants of the U2af1 gene were amplified by RT-PCR on RNA obtained from 11.5-day-old murine embryos using the following U2af1 sequence-specific primers: U2AF1FP: 5'-ATGGCGGAATACTTGGCCTCCATCTTCG-3'; U2AF1RP: 5'-TCAAAATCGTCCAGATCTCTCCCGGTC-3'.

PCR products were subsequently cloned into the pGEM-T easy vector (Promega) and sets of inserts from resulting clones were sequenced from both ends. The construct expressing the splicing variant U2af $1 b$ containing ORF was prepared by cloning it in frame into the pGADT7 vector.

\section{Cell culture and cell transfection}

Mouse fibroblast cells (NIH3T3) and the mouse spermatogoniaspecific cell line GC-1spg (Hofman et al., 1992) were obtained from the American Type Culture Collection (ATCC) (Rockville, USA) and maintained in DMEM growth medium (PAN) supplemented with 10\% fetal bovine serum (FBS) (PAN), $1 \times$ non-essential amino acids, $1 \%$ sodium pyruvate, $1 \%$ glutamine $(200 \mathrm{mM})$ and $10 \mu \mathrm{g} / \mathrm{ml}$ penicillin/ streptomycin. The spermatocyte-specific cell line GC-4spc was established and described previously (Tascou et al., 2000). Cell lines were incubated at $37^{\circ} \mathrm{C}$ and in an atmosphere of $5 \% \mathrm{CO}_{2}$. Transfections were performed with the PolyFect transfection reagent (Qiagen) according to the manufacturer's instructions.

\section{Cytochemical and immunohistochemical analyses}

NIH3T3 cells $\left(5 \times 10^{4}\right.$ cells) were plated on coverslips 24 h before transfection and washed with PBS. Construct DNA pEGFP/Phf5a-C1 or DsRed/Sfrs5-C1 (1.5 $\mu \mathrm{g}$ per well) was then introduced into the cells using the PolyFect transfection reagent (Qiagen) according to the man- ufacturer's instructions. Twenty-four hours later, transiently transfected cells were fixed on chamber slides with $4 \%$ paraformaldehyde for 15 min. If necessary, cells were stained with an antibody by blocking in $2 \%$ BSA solution in PBS buffer, subsequently incubated overnight at $4{ }^{\circ} \mathrm{C}$ with primary antibodies, and $2 \mathrm{~h}$ at room temperature with secondary, either FITC or Cy3-coupled, antibodies. After washing in PBT buffer, the stained and transiently transfected cells were mounted with Vectashield mounting medium with DAPI (Vector Laboratories Inc.) and observed under a fluorescence microscope (BX-60, Olympus).

For the immunostaining of cell suspensions, testes were isolated from adult male mice. The Tunica albuginea was removed, and testes were incubated in 10 volumes of Hank's balanced salt solution without $\mathrm{Ca}^{2+}$ and $\mathrm{Mg}^{2+}$ (HBSS), containing $1 \mathrm{mg} / \mathrm{ml}$ collagenase (Type IV, Sig$\mathrm{ma}$ ) and $200-500 \mu \mathrm{g} / \mathrm{ml} \mathrm{DNAse}$, at $37^{\circ} \mathrm{C}$ with gentle agitation for 15 min until tubules separated. Tubules were dispersed by careful dissection and washed 2-4 times in 10 volumes of HBSS and incubated in $1 \mathrm{mM}$ EDTA $/ 0.25 \%$ trypsin for $5 \mathrm{~min}$ at $37^{\circ} \mathrm{C}$. After separation and dispersion of tubular cells, 10-20\% FBS was added, and cells were filtered through a nylon mesh with $60 \mu \mathrm{m}$ pore size. The filtrate was centrifuged at $600 \mathrm{~g}$ for $5 \mathrm{~min}$ at $16^{\circ} \mathrm{C}$, and the supernatant was removed carefully from the pellet. Finally, cells were resuspended in the sperm cell medium or Dulbecco's modified Eagle's medium, containing 10\% FBS. Finally, cells were suspended $\left(4 \times 10^{7}\right.$ cells in $\left.8 \mathrm{ml} \mathrm{DMEM}+\mathrm{FBS}\right)$, divided among four coated dishes, and used for immunostaining.

Cryosections of mouse testes were fixed in ice cold isopropanol for $1 \mathrm{~min}$. Unspecific binding was blocked using 5\% BSA in PBS for $30 \mathrm{~min}$. After rinsing specimens in PBS, primary antibodies were diluted in PBS, 0.05\% BSA (rabbit anti-Phf5a 1:25; rabbit anti-U2af1 1:600, anti$\alpha$-tubulin 1:500) and incubated for $1 \mathrm{~h}$ at room temperature. After washing, the sections were decorated with anti-rabbit IgG conjugated with Cy3 (1:200) for $30 \mathrm{~min}$ in the dark and subsequently counterstained with DAPI. Coverslipped specimens were documented using a Leica fluorescence microscope.

\section{Western blotting}

The anti-PHF5A antibody was previously described by Trappe et al. (2002b). The anti-U2AF1 antibody was kindly provided by T. Maniatis and M. Carmo-Fonseca (Department of Molecular and Cellular Biology, Harvard University, Cambridge, MA, USA). $50 \mu$ g protein extracts were denatured with SDS sample buffer followed by 5 min boiling. Proteins were separated by electrophoresis using a NuPage SDS/4$12 \%$ Bis-Tris pre-cast polyacrylamide gel (Invitrogen) in MES buffer (Invitrogen) and transferred to a nylon Hybond-C membrane (Amersham). The resulting protein-bound membrane was blocked with $4 \%$ skim-milk and blotted with selected antibodies as described above. All Western blotting experiments were performed with the chemiluminescent substrate for detection of the HRP-coupled antibody using the SuperSignal West Pico Kit (Pierce) according to the original protocol.

\section{Results}

\section{Identification of PHF5A interaction partners}

To identify molecules that interact with PHF5A, we employed the yeast two-hybrid assay by using a murine 11.5day-old embryo library and screening with a $\mathrm{BD} / \mathrm{Phf5a}$ construct encoding the complete open reading frame (ORF) of $P h f 5 a$ as bait. Additional experiments excluded autoactivation of reporter genes by the PHF5A protein in the yeast strain AH109. In total, $1 \times 10^{8}$ yeast clones were screened, and approximately 160 positive yeast clones were obtained growing on high stringency SD plates in the absence of leucine, tryptophan, histidine, and adenine and exhibiting strong activation of $\alpha$-galactosidase.

DNA sequencing of 60 of these clones revealed that four genes were present multiple times as independent overlap- 
ping clones: U2af1 (coding for a $35 \mathrm{kDa}$ subunit of $\mathrm{U} 2 \mathrm{AF}$ ), Sfrs5 (also known as SRp40), $D d x 1$ (also known as $m D o m$ ino) and Ep400 (also known as p400). We selected the following clones for further analyses: clone no. 35 containing a cDNA fragment of the splicing factor U2af1 from nucleotide position 21-740 corresponding to the complete ORF, clone no. 94 with the shortest interacting cDNA fragment of the splicing factor Sfrs 5 from nucleotide position 659832 therefore spanning the $\mathrm{C}$-terminal region rich in arginine-serine residues (RS domain), clone no. 81 with the shortest interacting cDNA fragment of the $D d x 1$ gene coding for a DEAD (Asp-Glu-Ala-Asp) box polypeptide 1 (ATP-dependent RNA helicase $\mathrm{DEAD} / \mathrm{H}$ ) from nucleotide position 1660-2303, and clone no. 100 containing the shortest interacting cDNA fragment of the Ep400 gene encoding the E1A binding protein p400 (SWI2/SNF2-type ATPase/ helicase) from nucleotide position 1880-2516. Since clone no. 94 encoding the C-terminal RS domain of the SFRS5 protein was sufficient to maintain interaction with PHF5A, we also generated a construct encoding the RS domain of U2AF1. Yeast co-transformants with PHF5A as prey and the RS domain of U2AF1 as bait could grow on selective plates suggesting that PHF5A could represent an RS domain binding protein.

Double transformants of the bait-Phf5a construct and constructs containing library founders resulted in blue colonies as a consequence of the Mell gene activation. The product of the Mel1 gene, $\alpha$-galactosidase, could be quantitatively measured in the yeast liquid medium allowing direct comparison of the relative strength of interaction of PHF5A with different proteins.

First, in order to answer the question whether PHF5A interacts unspecifically with all RS proteins or if PHF5A binding is rather restricted to proteins found in our yeast two-hybrid library screening, RS domains of three other proteins were cloned and tested. The $\mathrm{BD} / \mathrm{Phf5a}$ bait construct was co-transformed together with constructs containing RS domains of the following splicing factors: Sfrs 1 (Cartegni and Krainer, 2002), Sfrs3 (Jumaa et al., 1997) and Sfrs9 (Park et al., 2006), and was subsequently tested on selective SD plates (-Leu, -Try, -His, -Ade). Yeast cells containing the bait and the Sfrs 9 construct could not grow on these plates, and co-transformants bearing the bait and Sfrs1 or Sfrs 3 could form colonies, but yeast cells did not turn blue in the presence of X-gal (data not shown). Second, we used a quantitative $\alpha$-galactosidase assay to compare the strength of PHF5A interaction with interaction partners previously identified in the yeast-two hybrid assay (Fig. 1A). Whereas galactosidase activity in yeast clones bearing PHF5A as a bait and RS domain of Sfrs1 or Sfrs 3 was almost undetectable, Ep400 and Sfrs5 showed moderate induction rates, and the strongest activity was measured for $D d x 1$ helicase and U2af1. Taken together, these results indicate that either there are no interactions between PHF5A and SFRS1, SFRS3, SFRS9 or the interactions were undetectable in this assay.
Co-immunoprecipitation of PHF5A with putative

interaction partners

Next, we studied whether in vitro protein-protein interactions would corroborate the yeast two-hybrid interactions. Therefore, new constructs containing HA-tagged versions of Sfrs5, U2af1, Ddx1 and Ep400 were prepared and recombinant proteins were produced by in vitro transcription-translations as described in the Materials and methods section. These HA-tagged proteins and their putative binding partner PHF5A (c-Myc-tagged) were used in the following co-immunoprecipitation assays.

After a 1-h incubation step at room temperature we could efficiently co-immunoprecipitate all four HA-tagged fusion proteins (SFRS5, U2AF1, DDX1 and EP400) with c-Myctagged PHF5A (Fig. 1B, C). These interactions were detectable in all cases using both combinations of either c-Myc or $\mathrm{HA}$ antibodies recognizing the adequate epitopes. Unspecific precipitation of HA-tagged proteins using the anti-cMyc antibody and c-Myc-tagged proteins using the anti-HA antibody, respectively, were not observed. In addition, the PHF5A-U2AF1 protein complex was even stable after extensive washing steps in a wide range of $\mathrm{NaCl}$ concentrations in the washing buffer (Fig. 1D), suggesting a hydrophobic rather than ionic interaction between PHF5A and the RS domain of U2AF1.

\section{Mapping of PHF5A interaction domains}

In order to map PHF5A interaction domains, several deletion constructs of mouse PHF5A were generated and tested for the ability to bind to the RS domain of U2AF1 by using an immunoprecipitation assay. For this experiment the C-terminal part of U2AF1 (U2AF1RS = U2AF1 fragment from amino acid position 173-239) containing the RS domain and an HA-tag was expressed using the construct $\mathrm{AD} /$ U2AF1-RS and co-immunoprecipitated by HA antibody using different protein segments of PHF5A. As can be seen in Fig. $2 \mathrm{~A}$, only the truncated protein fragment PHF5A $\Delta 81$ 110 could be effectively co-immunoprecipitated with the RS domain of U2AF1, whereas constructs PHF5A $\Delta 1-28,81-$ 110 and PHF5A $\Delta 1-28$ did not interact with U2AF1RS. This result suggests that the N-terminal part of PHF5A is responsible for the interaction with the RS domain of U2AF1. In this particular region of PHF5A two putative zinc fingers are localized which presumably fold into a ring finger domain (Oltra et al., 2003), thus mediating protein-protein interactions (Fig. 2B).

To determine more precisely the interaction domain of PHF5A with its interaction partners, we employed a directed yeast two-hybrid assay. For this study minimal interacting fragments of U2AF1, SFRS5, DDX1 and EP400 were used for co-transformations of yeast cells with different segments of PHF5A (Fig. 2B). RS domains of U2AF1 and SFRS5 were able to strongly associate with the deletion mutant PHF5A $\Delta 81-110$ and to a weaker extent with the deletion mutant PHF5A $\Delta 58-110$. Co-transformants bearing the RS domains and the isolated first two zinc fingers of PHF5A (PHF5A $\Delta 58-110$ ) had the ability to grow, but did not turn blue in the presence of $\mathrm{X}$-gal suggesting that an interaction 
A

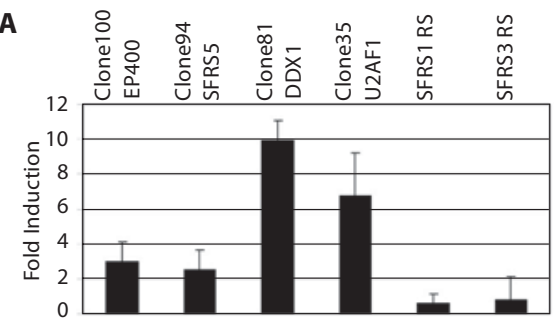

C

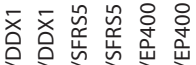
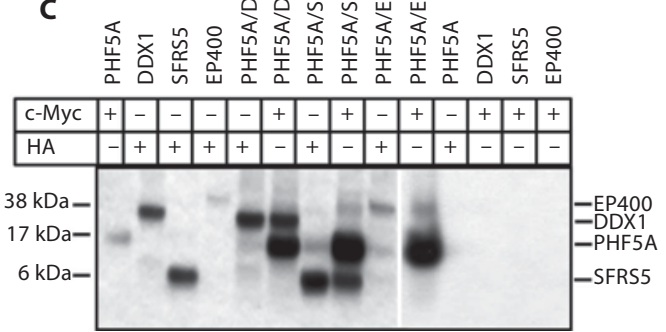

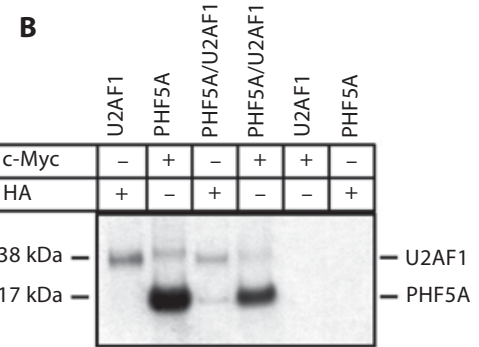

D

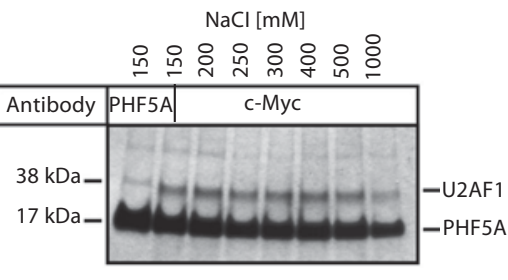

Fig. 1. Mouse PHF5A interacts with two SR proteins and two helicases. (A) The $\mathrm{X}$-gal quantitative assays show relative strength of interaction between mouse PHF5A and splicing proteins. The bars show the fold induction of $\mathrm{X}$-gal activity as compared to the empty vectors, error bars represent standard deviation, $N=3$. (B-D) SDS-PAGE analysis of co-immunoprecipitated proteins. Analyzed proteins were tagged with an $\mathrm{HA}$ or c-Myc epitope, respectively, and labeled with ${ }^{35} \mathrm{~S}$-methionine. After immunoprecipitation and elution, $10 \mu \mathrm{l}$ of the immunoprecipitate was loaded on to a $4-12 \%$ SDS polyacrylamide gel. In the description line the abbreviations of protein constructs are given; + or correspond to the antibody used for immunoprecipitation. (B) Co-immunoprecipitation of murine PHF5A and U2AF1. The in vitro transcription/translation was performed using the construct $\mathrm{BD} / \mathrm{Phf} 5 \mathrm{a}$ (c-Myc epitope) and AD/U2af1 (HA epitope) and ${ }^{35}$ S-labeled PHF5A$\mathrm{c}-\mathrm{Myc}$ and U2AF1-HA fusion proteins were generated. As a positive control protein from a single transfection was used. In the lane U2AF1 a $38-\mathrm{kDa}$ band was detected representing the U2AF1-HA fusion protein immunoprecipitated with the anti-HA antibody. Similarly, in the lane PHF5A the 17-kDa band represents the PHF5A-c-Myc fusion protein immunoprecipitated with an anti-c-Myc antibody (note unspecific band $>38 \mathrm{kDa}$ in this lane). In the lane PHF5A/U2AF1, in which proteins immunoprecipitated with the anti-HA antibody were loaded, besides the 38-kDa U2AF1-HA fusion protein band, the $17 \mathrm{kDa}$ PHF5A$\mathrm{c}-\mathrm{Myc}$ fusion protein band was also observed. In the next lane, in which proteins immunoprecipitated with the anti-c-Myc antibody were loaded, both the $17-\mathrm{kDa}$ PHF5A-c-Myc fusion protein band and the $38 \mathrm{kDa}$ band representing the U2AF1-HA fusion protein were detected. As

with the first three fingers of PHF5A is preferred. In contrast, the results of the interaction studies with both DDX1 and EP400 demonstrated that both helicases could associate with the C-terminal part of PHF5A. A minimal domain present in all fragments of PHF5A interacting with helicases was identified which could be narrowed down to the third zinc finger of PHF5A.

\section{PHF5A represents a bridge protein between SR proteins and helicases}

Our previous results showed that the N-terminal part of PHF5A functions as the binding site for the RS domains of splicing factors and that the third zinc finger of PHF5A represents the interacting part responsible for binding to heli- negative control the HA-tagged protein was immunoprecipitated with the anti-c-Myc antibody and the c-Myc-tagged protein was immunoprecipitated with the anti-HA antibody, respectively (last two lanes). (C) Co-immunoprecipitation of murine PHF5A with DDX1, SFRS5 and EP400. The c-Myc tagged PHF5A fusion protein was generated as described above and the HA tagged fusion proteins were generated from constructs $\mathrm{AD} / \mathrm{DDX} 1-\mathrm{RS}, \mathrm{AD} / \mathrm{SFRS} 5-\mathrm{RS}$, and AD/EP400-RS, respectively. As positive control proteins from cells transfected with single constructs were precipitated with an antibody corresponding to the tag (first four lanes: PHF5A, DDX1m, SFRS5 and EP400). In the lane PHF5A/DDX1, containing proteins immunoprecipitated with the antiHA antibody besides the strong DDX1-HA fusion protein band, an additional PHF5A-c-Myc band could be detected. Similarly, in the next lane, where proteins were immunoprecipitated with the anti-c-Myc antibody besides the PHF5A-c-Myc band, the DDX1-HA band was visible. Co-immunoprecipitations were detectable in all cases using both antibody combinations. Unspecific precipitations with opposite antibodies were not detected (last four lanes). (D) SDS-PAGE analysis revealed that interaction between PHF5A and U2AF1 examined by immunoprecipitation is salt-independent. As a control the PHF5A-c-Myc fusion protein was immunoprecipitated with an anti-PHF5A antibody (first lane). Subsequently, the PHF5A/U2AF1 complex was precipitated with an anti-c-Myc antibody in an increasing gradient of $\mathrm{NaCl}$ concentrations.

cases. In order to determine whether PHF5A was able to bind SR proteins and helicases simultaneously, we employed the yeast three-hybrid assay. Yeast cells were transformed with a bi-cistronic pBridge vector either expressing U2AF1 or SFRS5 constitutively as a binding domain (BD) fusion. The second protein, i.e. PHF5A, was expressed under the control of the conditional $\mathrm{P}^{\mathrm{Met} 25}$ promoter which is only active in yeast cells growing on selective SD plates in the absence of methionine. Furthermore, pBridge constructs were used in conjunction with pGADT7 constructs expressing either DDX1 or EP400 as an activation domain (AD) fusion. For all constructs analyzed, a continuous growth of double transformed yeast cells was observed on SD plates lacking methionine, tryptophan, leucine, adenine and histidine, 
Fig. 2. PHF5A is a bridge protein between SR proteins and helicases. (A) SDS-PAGE analysis showed that the truncated murine PHF5A protein containing the $\mathrm{N}$-terminal part interacts with the arginineserine (RS)-rich domain of U2AF1. In vitro transcribed/translated fusion proteins U2AF1-RS-HA, PHF5A $\Delta 81-110$, PHF5A $\Delta 1-28,81-110$, PHF5A $\Delta 1-28$, were generated from constructs AD/U2af1-RS, GST/ Phf5a $\Delta 1-28$, GST/Phf5a $\Delta 1-28,81-110$, GST/Phf5a $\Delta 1-28$, respectively, and labeled with ${ }^{35} \mathrm{~S}$-methionine. $10 \mu \mathrm{l}$ of the HA antibody immunoprecipitate was loaded onto $4-12 \%$ SDS polyacrylamide gel. In the description line the abbreviation of each protein construct is given. The first lane (U2AF1RS) represents the fusion protein band from a single transfection with the HA-tagged RS domain of the U2AF1 protein immunoprecipitated with the anti-HA antibody. In the next lane (U2AF1RS/PHF5A $\Delta 81-110$ ), besides the U2AF1RS band, an additional band was detected which represents co-immunoprecipitated truncated PHF5A protein containing amino acids $81-110$. In contrast, coimmunoprecipitation of other truncated PHF5A protein forms (Phf5a $\Delta 1-28,81-110, \operatorname{Phf5a} \Delta 1-28)$ with the RS domain of U2AF1 was not observed (next two lanes). As a negative control in vitro transcribed/translated proteins PHF5A $\Delta 81-110-H A, P H F 5 A \Delta 1-28,81-$ $110-\mathrm{HA}$ and PHF5A $\Delta 1-28$-HA were loaded in the three last lanes, respectively. (B) Schematic view of the truncated fragments of PHF5A used for mapping of interaction domains in the immunoprecipitation experiment as well as in a direct yeast two-hybrid assay. Protein interactions with different segments of PHF5A, which were analyzed by the yeast-two hybrid assay, are summarized in the table. The $\mathrm{N}$-terminal part of PHF5A is responsible for the interaction with U2AF1 and SFRS5 and the $\mathrm{C}$-terminal part is required for the interaction with DDX1 and EP400. Abbreviations: finger 1 and finger 2: zinc finger no. 1 and zinc finger no. 2 composing the RING domain; finger 3: zinc finger no. 3; L-zipper: leucine zipper. (C) Yeast cells transformed with a pBridge construct containing the Phf5 $a$ sequence and pGADT7 constructs containing U2af1, Sfrs5, Ddxl and Ep400 sequences were either streaked on a selective SD plate without methionine (-Met, -Leu, -Try, -Ade, -His; left) or on a selective SD plate containing $1 \mathrm{mM}$ methionine (-Leu, -Try, -Ade, -His; right) and analyzed for cell growth. The explanations for the numbers of the plate quarters are given in the table. $\mathrm{BD}$ : binding domain; AD: activation domain.

while the addition of methionine at a concentration of $1 \mathrm{mM}$ could almost arrest the growth of yeast cells (Fig. 2C). These results strongly indicate that PHF5A indeed acts as a bridge protein between splicing factors U2AF1 and SFRS5 and ATP-dependent helicases DDX1 and EP400.

\section{Characterization of U2af1 splicing forms}

By using the yeast two-hybrid screen, we identified one clone corresponding to a shortened form of the U2af1 mRNA, which prompted us to analyze alternative splicing forms of this gene. The genomic organization of the mouse U2af1 gene was obtained from the ENSEMBL data base (www.ensembl.org) and is schematically represented in Fig. 3A. The genomic U2af1 sequence was blasted against the sequence of the isolated shortened form of U2af1 mRNA showing that this clone lacked both exon 2 and 3 sequences (Fig. 3A, U2af1d). This U2af1 splicing form contains a premature internal stop codon in exon 5 as a result of a frame shift. To examine the presence of other splicing forms of the U2af1 gene, we performed RT-PCR experiments on RNA isolated from 11.5-day-old mouse embryos using primers U2AF1FP and U2AF1RP amplifying the complete open reading frame (ORF) of U2af1. Subsequent sequence analy-
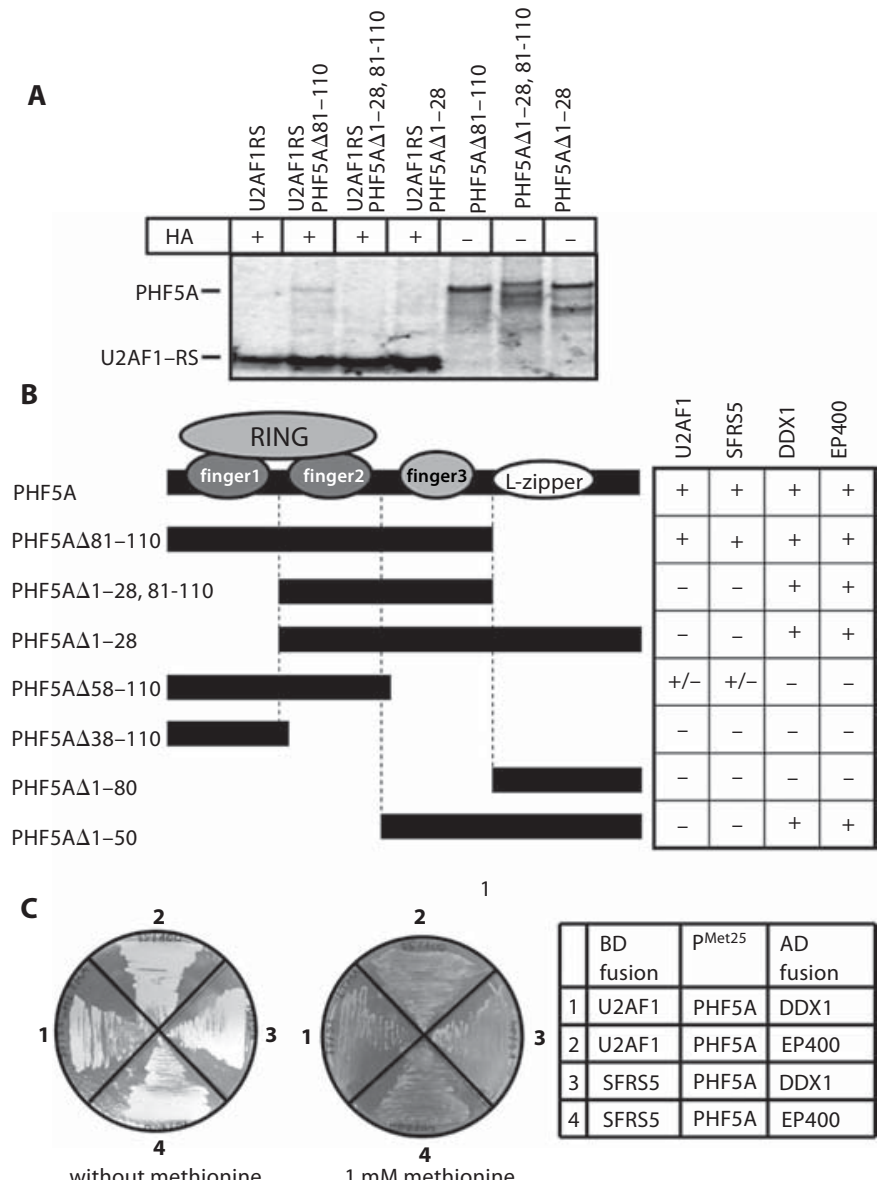

without methionine

$1 \mathrm{mM}$ methionine

ses revealed the presence of one additional U2af1 splicing form with a missing exon 3 sequence, which is characterized by a shortened ORF leading to a truncated U2af1 transcript (Fig. 3A, U2af1c). Moreover, a third fragment was identified corresponding to a recently described splicing form of U2af 1 (Pacheco et al., 2004) where exon 3 is substituted by an alternative exon $\mathrm{Ab}$ (Fig. 3A, U2af1b). The U2af1b splicing form contains the full ORF, and the translation of this alternative mRNA leads to a change of only seven amino acids. The U2af1 splicing variants were grouped (Fig. 3A) and assigned as: U2af1a corresponding to the previously described form (Zhang et al., 1992); U2af1b which is identical to human splicing pattern $b$, where exon 3 is substituted by exon Ab (Pacheco et al., 2004); U2af1c with a missing exon 3 sequence and U2afld lacking exon 2 and 3 sequences.

A further co-immunoprecipitation experiment was performed in order to determine whether the alternative variant U2AF1b shows an identical binding affinity to PHF5A as compared to the variants U2AF1a and U2AF $\Delta 1-67$. The $\mathrm{U} 2 \mathrm{AF} \Delta 1-67$ variant bears a deletion in its $\mathrm{N}$-terminal part and contains the sequence downstream of exon 3. Because the $\mathrm{N}$-terminal region of the U2AF1 protein is known to be responsible for the association with U2af2 (Zhang et al., 


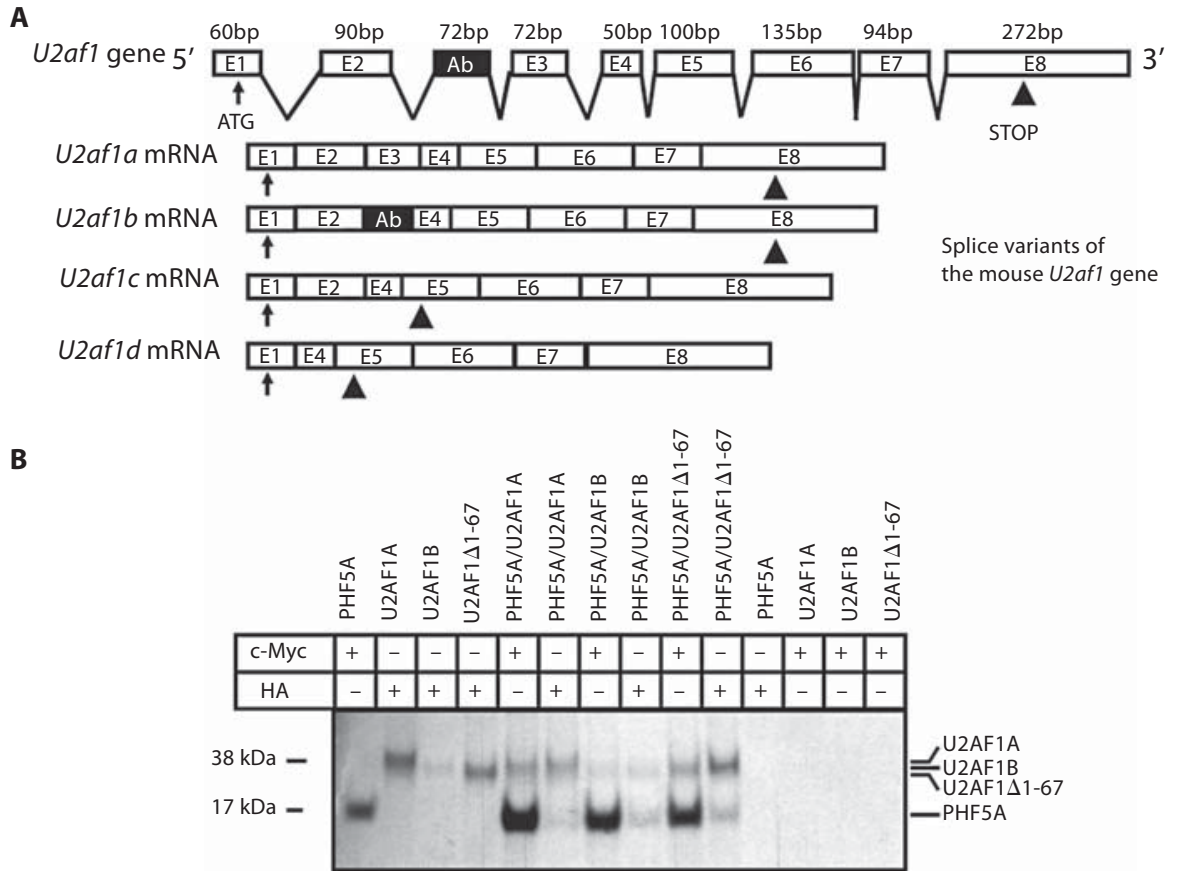

Fig. 3. Alternative splicing of the U2af1 gene and interaction with PHF5A. (A) Schematic representation of the genomic organization of the murine U2af1 gene and different splicing variants for U2af1 detected by using RT-PCR experiments. Exons are represented by boxes and numbers, the start codon is indicated by an arrow and the stop codon is presented by an arrowhead. (B) SDS-PAGE analysis demonstrating that murine PHF5A co-immunoprecipitates with different splicing forms, namely U2AF1A, U2AF1B and U2AF1 $1-67$ (spanning the sequence from exon 4 to the stop codon). In vitro transcribed and radioactively labeled ${ }^{35} \mathrm{~S}$-proteins PHF5A, U2AF1A, U2AF1B and U2AF1 $\Delta 1-67$ were generated from constructs BD/Phf5a (c-Myc epitope), $\mathrm{AD} / \mathrm{U} 2 \mathrm{afla}$ (HA epitope), $\mathrm{AD} / \mathrm{U} 2 \mathrm{af1b}$ (HA epitope) and $\mathrm{AD} /$ U2af1 $\Delta 1-67$ (HA epitope), respectively. Generated proteins were used in the immunoprecipitation experiments with either anti-c-Myc or

1992), we assumed that this part does not interact with PHF5A. The constructs of the U2AF1 variants were expressed as HA-tagged fusion proteins and used in co-immunoprecipitation assays together with a c-Myc-tagged PHF5A fusion protein (Fig. 3B). All variants of U2AF1 could be efficiently co-immunoprecipitated with PHF5A indicating that a modification in the N-terminal part of U2AF1 does not influence the binding properties between U2AF1 and PHF5A.

\section{Subcellular co-localization of PHF5A with U2AF1 and SFRS5}

Next, the subcellular distribution of mouse PHF5A-GFP fusion protein together with splicing proteins U2AF1 and SFRS5 was analyzed in mouse NIH3T3 cells by immunocytochemical studies (Fig. 4). NIH3T3 cells were transiently transfected with a construct expressing a PHF5A-GFP fusion protein and subsequently stained with an anti-U2AF1 antibody and an anti-rabbit-Cy 3 conjugated secondary antibody. Signals from the green PHF5A fusion protein and from the endogenous U2AF1 protein (red) partially co-lo-
anti-HA antibody and separated on an SDS polyacrylamide gel. In the description line the abbreviations of protein constructs and antibodies used are given. In the first four lanes (PHF5A, U2AF1A, U2AF1B and $\mathrm{U} 2 \mathrm{AF} 1 \Delta 1-67)$ labeled fusion proteins from single transfections are loaded as positive controls. In the lane PHF5A/U2AF1A, which contains proteins immunoprecipitated with an anti-c-Myc antibody besides the PHF5A band (17 kDa), also the U2AF1A band ( $38 \mathrm{kDa})$ was detected. Likewise, in the next lane proteins were loaded after immunoprecipitation with an anti-HA antibody and besides the U2AF1A band $(38 \mathrm{kDa})$ also the PHF5A band $(17 \mathrm{kDa})$ was observed. In the next two lanes, both other tested splice variants (U2AF1B and U2AF1 $\Delta 1-$ 67) could also be efficiently co-immunoprecipitated with the PHF5A protein. The last four lines represent negative controls (proteins immunoprecipitated with improper antibodies).

calized and were distributed throughout the nucleus. Signals for both PHF5A and U2AF1 were not observed in nucleoli, but were enriched in multiple speckles in the nucleus (Fig. 4A-D). Double transfection of NIH3T3 cells with vectors expressing PHF5A-GFP and SFRS5-DsRed fusion proteins revealed that PHF5A could also be co-localized with the second interacting partner in a pattern restricted to speckled regions in the nucleus (Fig. 4E-H).

\section{Expression and localization of PHF5A and U2AF1 in mouse testis}

Immunofluorescence staining of mouse testis sections using a rabbit anti-PHF5A antibody revealed that this protein is mainly confined to the nuclei of primary spermatocytes (Fig. 5A, E). The strongest signal was observed in pachytene spermatocytes, but earlier meiotic cells such as leptotene and zygotene spermatocytes were also positive. Again, nuclear staining of PHF5A was in a speckled fashion. PHF5A immunoreactivity was very weak in some scattered cells, which can not represent the entity of Leydig cells. 

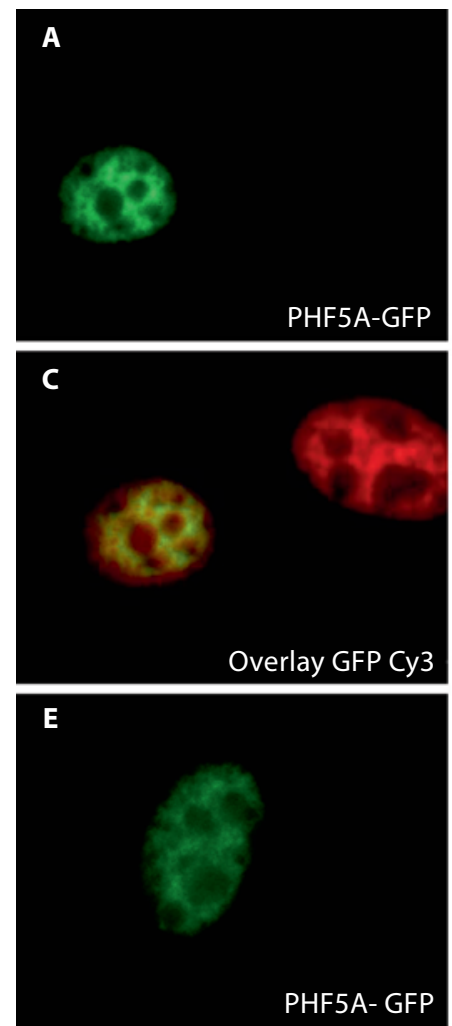

G

4

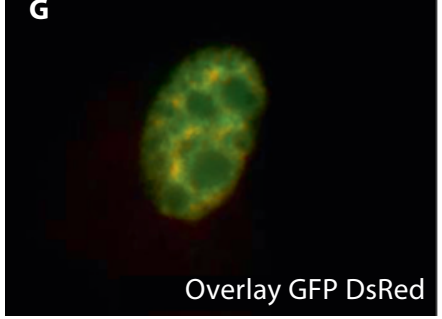

H

SFRS5-DsRed
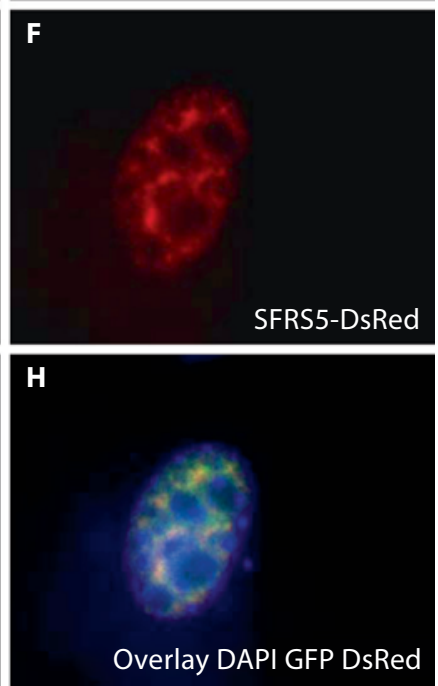

Fig. 4. Sub-cellular co-localization of PHF5A with splicing proteins U2AF1 and SFRS5 in nuclear speckles of NIH3T3 cells. (A-D) The PHF5A-GFP fusion protein could be partially co-localized in the nucleus of NIH3T3 cells with U2AF1 stained with rabbit anti-U2AF1 and anti-rabbit Cy3. (E-H) The PHF5A-GFP fusion protein shows partial co-localization with SFRS5-DsRed fusion protein in the nucleus of NIH3T3 cells. Nuclei of NIH3T3 cells were counterstained with DAPI. Magnification: $200 \times$.

Fig. 5. Immunofluorescence localization of PHF5A and U2AF1 in mouse testis. (A, E) The strongest PHF5A immunoreactivity is visible in nuclei of primary spermatocytes and a few scattered cells in the interstitium. (C, G) U2AF1 immunofluorescence is detectable in the nuclei of primary spermatocytes, round and some elongating spermatids. Spermatozoa are negative. Higher magnification $(\mathbf{E}, \mathbf{G})$ shows that the immunoreaction for PHF5A appeared in multiple punctuate sites in the nuclei of primary spermatocytes, whereas U2AF1 labeling in nuclei of spermatids concentrated into few, but larger foci (G). (B, D, F, H) corresponding DAPI staining.

Fig. 6. Immunostaining of testicular cell suspension with antiPHF5A and anti- $\alpha$-tubulin antibodies. (A) Staining with rabbit antiPHF5A and anti-rabbit Cy3 antibody. (B) Staining with mouse anti- $\alpha$ tubulin and anti-mouse FITC conjugated antibody. (C) DAPI staining. (D) Overlay of DAPI, FITC and Cy3 staining. The strongest PHF5A expression was detected in nuclei of spermatocytes, but also weak signals from site accumulations of PHF5A were observed in spermatids (marked with an arrow). spc, spermatocytes; spd, spermatids.
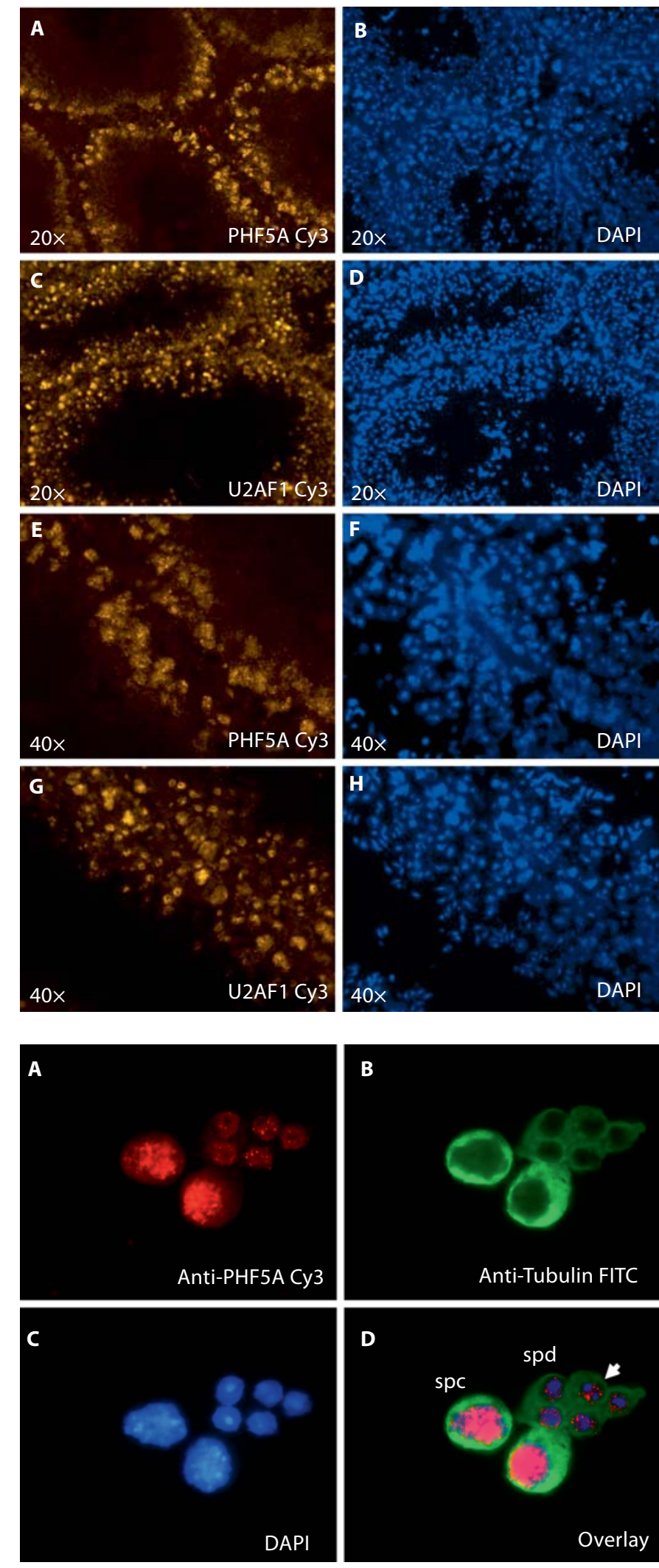

6 
A

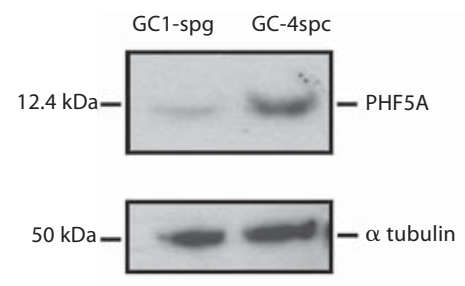

C

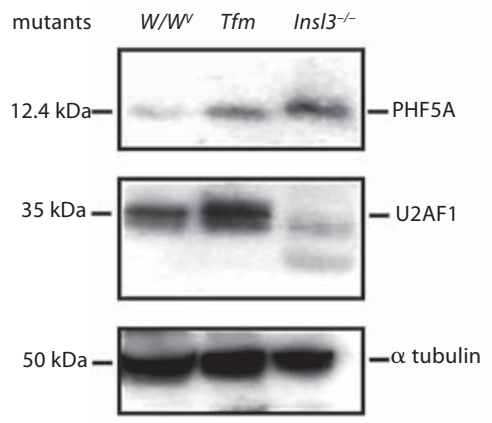

B $\quad \begin{array}{lllllll}\text { dpp } & 5 & 10 & 12 & 16 & 25 & \text { Adult }\end{array}$

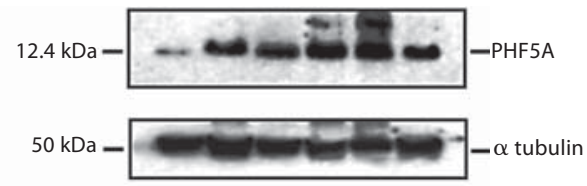

$\begin{array}{lllllllll}\text { dpp } & 5 & 10 & 12 & 16 & 19 & 21 & 25 & 40\end{array}$

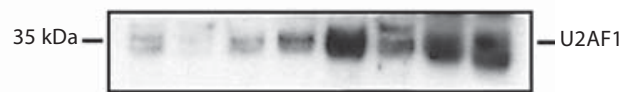

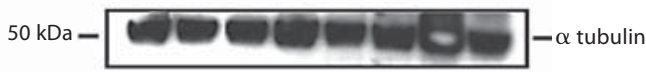

D

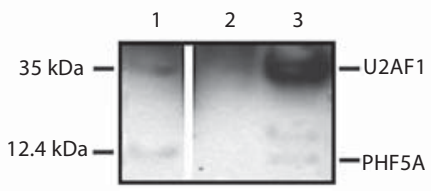

Fig. 7. Expression and interaction analyses of PHF5A and U2AF1 during mouse spermatogenesis. (A) Western blot showing that PHF5A expression is up-regulated in GC-4spc cells as compared to GC-1spg cells. Proteins were separated on $4-12 \%$ SDS polyacrylamide gel, transferred onto a nylon membrane and incubated with anti-PHF5A and anti- $\alpha$ tubulin antibodies, respectively. (B) Western blots showing that both PHF5A and U2AF1 expression increase during postnatal testis development. Protein extracts from testis at different days post partum (dpp) $(5,10,12,16,25$ and from an adult animal) were separated and probed with an anti-PHF5A antibody. Proteins from mouse testis at 5, $10,12,16,19,21,25$ and 40 dpp were probed with an anti-U2AF1 antibody. As a loading control an anti- $\alpha$ tubulin antibody was used.
(C) Western blots showing expression of U2AF1 and PHF5A in testis of the mouse mutants $W / W^{\nu}, T f m$ and $I n s / 3^{-/}$. (D) SDS-PAGE analysis shows that PHF5A co-immunoprecipitates with U2AF1 in GC-4spc protein extract. Cell lysate from GC-4spc cells was incubated with an anti-PHF5A antibody and subsequently immunoprecipitates were loaded (lane 1). The membrane was incubated with both anti-PHF5A and anti-U2AF1 antibodies. Signals for both PHF5A (12.4 kDa) and U2AF1 $(35 \mathrm{kDa})$ could be detected. In lane $2 \mathrm{GC}-4$ spc cell protein extract, incubated with A beads only, was loaded (without anti-PHF5A antibody, negative control) and lane 3 contains protein extract from GC-4spc cells (not immunoprecipitated, positive control).
The expression of U2AF1 was also examined as the binding studies suggested at least a partial temporospatial overlap with PHF5A synthesis in testis. U2AF1 was detectable in all types of spermatocytes as well as in round and some elongating spermatids, but not in spermatozoa (Fig. 5C, G). The strongest fluorescence signal for both proteins was clearly evident in pachytene spermatocytes. Nuclei of cells are visualized with DAPI staining (Fig. 5B, D, F, H).

Interestingly, the PHF5A nuclear immunoreactivity pattern is dynamic during progression of mouse spermatogenesis. As demonstrated on testicular cell suspension stained with anti-PHF5A antibody, multiple punctuate sites were observed in nuclei of primary spermatocytes, whereas only few but extended positive foci could be seen within the nuclei of round spermatids (Fig. 6). The low intensity of the PHF5A staining in spermatid suspensions could be a reason why PHF5A signals in spermatids could not be detected in mouse testis sections (Fig. 5).

Subsequent Western blot analysis revealed that the expression of PHF5A differs between the spermatogonia-derived GC-1spg cell line and the spermatocyte-specific cell line GC-4spc (Fig. 7A). This result is in accordance with our previous findings for PHF5A expression at the mRNA level (Trappe et al., 2002a). As demonstrated in Fig. 7B the expression of PHF5A increased during postnatal testicular development. Only a weak signal of PHF5A was found in the protein extract isolated from testis of 5-day-old mice, where spermatocytes are still absent (Silver, 1995) (Fig. 7B). Expression of PHF5A was noticeably higher starting from ten days post partum (dpp) when early spermatocytes appear in the testis (Silver, 1995). In testes of both the Tfm and $I n s l 3^{-/-}$mutant mice, which are characterized by an arrest of spermatogenesis at the spermatocyte level (Lyon and Searle, 1989; Zimmermann et al., 1999), PHF5A expression also increased noticeably as compared to testicular expression in the $W / W^{\nu}$ mutant mouse (Fig. 7C) that lacks germ cells (Lyon and Searle, 1989). The latter result supports the observation that PHF5A was also found to be weakly expressed in somatic (interstitial) cells on testis sections.

The U2AF1 protein expression in the postnatal mouse testis could be observed starting already at $5 \mathrm{dpp}$, however, highest U2AF1 expression was found between 16 and $19 \mathrm{dpp}$ (Fig. 7B). Surprisingly, expression of U2AF1 in the testis of 
the Insl3 ${ }^{-/-}$mutant is highly decreased in comparison to PHF5A expression in testes of $T f m$ and $W / W^{\nu}$ mutant mice (Fig. 7C).

\section{Immunoprecipitation of PHF5A and U2AF1 in} GC-4scp cells

The results obtained from immunostaining experiments on testis sections revealed that the highest expression rates of both PHF5A and U2AF1 proteins were detectable in pachytene spermatocytes. Based on this result, we selected the murine spermatocyte-derived GC-4spc cell line to verify that the interaction between PHF5A and U2AF1 could also exist in vivo. Using an anti-PHF5A antibody we could efficiently co-precipitate U2AF1 protein from the GC-4spc cell extract (Fig. 7D). Both interaction partners were subsequently visualized by blotting the membrane with both anti-U2AF1 and anti-PHF5A antibodies. This result provides valuable evidence that an interaction between PHF5A and U2AF1 indeed exists in vivo.

\section{Discussion}

PHF5A is implicated in the processing of pre-mRNAs. By using a combination of yeast two-hybrid, immunoprecipitation, and immunohistochemical experiments, we could demonstrate that mouse PHF5A is associated with two SR proteins, namely U2AF1 and SFRS5, as well as with helicases DDX1 and EP400. These PHF5A interaction partners consist of either domains characteristic for RNA processing or were described to be involved in this process. Splicing factors SFRS5 and U2AF1 contain domains rich in alternating arginine and serine residues (RS domains). DDX1 and EP400 are ATP-dependent helicases and DDX1 is known to be involved in RNA processing (Godbout and Squire, 1993; Bleoo et al., 2001). EP400 was demonstrated to regulate the expression of developmentally essential genes located in the murine Hox gene cluster (Ueda et al., 2007).

The U2AF1 protein is part of the U2AF-heterodimer which is required for the proper recognition of the $3^{\prime}$ splicing site by the U2 snRNP (Ruskin et al., 1988) and while the large subunit $\mathrm{U} 2 \mathrm{AF} 2$ of this complex binds directly to the polypyrimidine tract (Zamore et al., 1992), the small U2AF1 subunit recognizes the AG dinucleotide at the 3' splicing site (Merendino et al., 1999; Wu et al., 1999). The RS domain localized in the C-terminal part of U2AF1 establishes protein-protein interactions with SR proteins (Wu and Maniatis, 1993), and it was proposed that SR proteins bind to the purine rich exonic splicing enhancers (ESE). This binding would facilitate $3^{\prime}$ splicing recognition of a weak pyrimidine tract by the large subunit U2AF2. U2AF1 may then act as a bridge protein and bind simultaneously to the SR proteins (Zuo and Maniatis, 1996). However, this mechanism has been argued by Guth et al. (2001) suggesting additional functions for U2AF1. The human SFRS5 protein was detected in the nucleus of different cells, and its role in the splicing process was proposed (Snow et al., 1997).
In the present study we could demonstrate that mouse PHF5A recognized specifically the RS domain of both U2AF1 and SFRS5 and showed low affinity to the RS domains of SFRS1 and SFRS3. Results from previous studies indicate that substrate specificity of SR proteins is determined by RNA recognition motifs (RRMs) and that the RS domains may have redundant functions in constitutive splicing (Chandler et al., 1997; Mayeda et al., 1999). Our results implicate that specificity of protein interaction with PHF5A is a function of individual serine-arginine rich domains. A minimal fragment of PHF5A able to interact with the RS domain of U2AF1 and SFRS5 is restricted to the N-terminal part of the protein. A deletion mutant bearing all three putative zinc fingers of PHF5A was sufficient to associate with RS domains in the yeast two-hybrid assay, whereas a deletion mutant with only two N-terminal zinc fingers showed weak affinity to the RS domain. It should be pointed out that deletion of either the first or the second zinc finger of PHF5A completely abolished PHF5A binding to the RS domains of U2AF1 or SFRS5. All three zinc fingers of rat PHF5A have been previously shown to be required for the activation of the estrogen receptor $\alpha(\mathrm{ER} \alpha)$ (Oltra et al., 2003).

PHF5A appeared also to associate with two proteins from the DEAD-like superfamily. Whereas DDX1 is an ATP-dependent RNA helicase (Godbout and Squire, 1993; Bleoo et al., 2001), EP400 seems to be part of the ATP-dependent chromatin-remodeling complex and belongs to the subfamily of SWI2/SNF2-type helicases (Ogawa et al., 2003). Members of this family have been isolated in association with the RNA polymerase II holoenzyme complex (Wilson et al., 1996; Neish et al., 1998). The role of helicases in RNA processing is reviewed by Bleichert and Baserga (2007).

Several lines of evidence indicate that chromatin remodeling and pre-mRNA splicing occur in coordination with transcription. PRP4 kinase was described as a protein interacting with splicing factors PRP6 and SWAP, and PRP4 was also shown to be associated with BRG1 and N-CoR, components of large multiprotein complexes involved in gene regulation mediated by nuclear hormone receptors (Dellaire et al., 2002). Another example of a tight association of transcription and splicing was demonstrated for the TLS protein, which interacted with SR proteins and whose N-terminal part is involved in transcriptional activation (Meissner et al., 2003).

Mapping experiments demonstrated that both DDX1 and EP400 interacted with the middle segment of PHF5A which can be more precisely restricted to the third zinc finger. Previously, this domain has been described as a fragment capable of binding to the promoter of the connexin 43 gene (Oltra and Werner, 1998; Oltra et al., 2003). Taken together, mouse PHF5A seems to possess two distinct domains for binding to SR proteins and ATP-dependent helicases. Using a yeast three-hybrid assay we could demonstrate that PHF5A is able to bind SR proteins and helicases simultaneously, most likely playing a role as a bridge protein. A large group of ATP-dependent helicases containing an RS domain was shown to directly interact with SR proteins. In contrast, DDX1 and EP400 do not interact directly 
and seem to interact with SR proteins only indirectly by using PHF5A as a bridge protein. In this context, a functional link between chromatin activation and splicing would exist via PHF5A.

New insights into the putative role of $P h f 5 a$ were presented by a study of Will et al. (2002), where the human homologous protein PHF5A (also known as INI or SF3b14b), apparently associated with a 17S U2 snRNP subunit named SF3b. This study demonstrated that human PHF5A could also play a role in splicing processes. Additional evidence that this protein associates with U2 snRNP proteins was presented by Wang and Rymond (2003). In this study, the homologous yeast protein RDS3P was found to be an interaction partner of U2 snRNP proteins, namely CUS1P, HSH49P, HSH155P, RSE1P, IST3P/SNU17P and the YRA1P RNA export factor. Cus1p, Hsh49p, Hsh155p and Rselp are known to encode components of yeast U2 snRNP (Wang and Rymond, 2003). Moreover, an Rds3p mutation caused disorganization of the U2 snRNP complex. In conclusion, PHF5A has been linked to different aspects and levels of gene expression, suggesting a putative role as a transcription factor and a protein involved in pre-mRNA splicing. This is strongly supported by the fact that the PHF5A sequence is highly conserved within vertebrates, but no ortholog of Phf5a could be found in the genome of prokaryotic organisms (Oltra et al., 2003).

$\mathrm{U} 2 \mathrm{AF}$ proteins are highly conserved during evolution, and in the human genome a number of $U 2 A F$-related genes are present. We could demonstrate that the mouse U2af1 gene undergoes alternative splicing and produces four different mRNA variants. Pacheco et al. (2004) have reported that human $U 2 A F 1$ is alternatively spliced resulting in three different mRNA variants. It should be noted that the human $U 2 A F 1 C$ splice variant is different from the splice form which we detected in the mouse. In human this variant contains all exons, including the alternative exon $\mathrm{Ab}$, whereas in the present study we could not observe such a variant in the mouse. Alternative splicing is rather a common event in $U 2 A F$-related genes in human (Mollet et al., 2006). Furthermore, the second SR protein coding gene Sfsr5 was also shown to undergo alternative splicing (Du and Taub, 1997).

The sub-cellular localization of PHF5A together with the partial co-localization of U2AF1 and SFSR5 in the nucleus of NIH3T3 cells is similar to that known for other splicing proteins (Abdelhaleem, 2005). The observed granular/ punctuate pattern presumably represents perichromatin fibrilles (PFs), which occur mainly at the periphery of condensed chromatin regions. These speckled regions represent the major nucleoplasmic ribonucleoprotein constituents rich in different splicing components (Bleoo et al., 2001). For U2AF1 and SFSR5 it was previously shown that both proteins are localized in nuclear speckles (Snow et al., 1997; Chusainow et al., 2005).

Very little is known about the mechanisms regulating pre-mRNA processing during development and maturation of male germ cells in mammals (Venables and Eperon, 1999). Transition from diploid spermatogonia to functional haploid sperm cells is accompanied by substantial changes in strategies for regulating gene expression, with a marked increase in post-transcriptional regulation (Braun, 1998; Steger, 2001). Alternative splicing regulation is critically important during spermatogenesis (Snow et al., 1997; Chusainow et al., 2005), and Phf5a could be a candidate gene for a factor taking part in this unique process.

Our observation that PHF5A is strongly expressed in the spermatocyte-specific cell line GC-4spc is consistent with the results of the immunolocalization and cell suspension studies. In male germ cell suspensions we could also demonstrate weaker expression of PHF5A in round spermatids. According to our expression studies, we can conclude that PHF5A is weakly expressed in pre-meiotic germ cells as well as in somatic cells of the testis, because we detected weak signals for PHF5A in the spermatogonia-specific cell line GC-1spg and in the testis of $W / W^{V}$ mutants. We could also demonstrate that PHF5A is co-expressed with U2AF1 predominantly in pachytene spermatocytes and both proteins showed a similar sub-cellular localization pattern in the nuclei of these germ cells. To our knowledge, our study represents the first report about U2AF1 expression in murine male germ cells. This finding is supported by the UniGene entry Mm.379289 where four testicular EST clones for murine U2af1 are reported (http://www.ncbi.nlm.nih.gov/sites/ entrez?db=unigene). Further UniGene analysis revealed that also other PHF5A interaction partners are presumably expressed in mouse male germ cells. There are 14 testicular EST clones corresponding to the Sfrs5 gene (UniGene entry Mm.43331), 19 testicular EST clones corresponding to the $D d x 1$ gene (UniGene entry Mm.251255), and 41 testicular EST clones representing the sequence of Ep400 (UniGene entry Mm.270487). Furthermore, expression of $D d x 4$, the mouse homologue of the Drosophila vasa gene, is restricted to germ cells (Leroy et al., 1989). In addition, another DDX gene family member, namely $D D X 3 Y$, is known to be essential for proper spermatogenesis in human (Foresta et al., 2000).

The results obtained in the present study raise the possibility that PHF5A, U2AF1 and other interaction partners of PHF5A take part in the meiotic differentiation of male germ cells. Rearrangements in the PFH5A localization in later germ cell stages are similar to rearrangements found by other studies (Moussa et al., 1994). During spermatogenesis both snRNPs (snRNAs and associated proteins) and non-snRNP components of the spliceosome undergo spatial reorganizations, and the RBM protein undergoes dynamic modulations in its spatial localization between different cell types (Elliott et al., 1998). It was described that during spermatogenesis the peak for gene expression is found at the spermatocyte stage, and then the global expression level decreases while haploid round spermatids differentiate into transcriptionally quiescent elongated spermatids (Monesi, 1964; Erickson, 1990). Therefore, it can be assumed that proteins involved in the splicing process are concentrated in discrete regions in the nucleus of round spermatids due to these global changes (Elliott et al., 1998). For PHF5A changes in sub-cellular localization may correlate with the reorganization of such splicing proteins. Finally, these changes 
resulted in few punctuated foci being the storage place for proteins involved in mRNA processing. Such a global rearrangement in the localization of snRNPs was also described by Antoniou et al. (1993) for murine erythro-leukaemia cells after induction of differentiation and in neuronal differentiation in the rat (Santama et al., 1996). Widespread nuclear snRNPs in later stages of differentiation disassociate from coil bodies and aggregate in foci corresponding to interchromatin granules.

In conclusion, the results of the present study indicate that PHF5A acts as a bridge factor between proteins of the splicing machinery and ATP-dependent helicases. In addi- tion, PHF5A was found to be strongly co-expressed with the splicing factor U2AF1 at the spermatocyte stage during mouse spermatogenesis, thus representing a potentially important factor for proper meiotic differentiation of male germ cells.

\section{Acknowledgment}

The authors wish to thank Wolfgang Engel for supporting this work in his institute.

\section{References}

Abdelhaleem M: RNA helicases: regulators of differentiation. Clin Biochem 38:499-503 (2005).

-Antoniou M, Carmo-Fonseca M, Ferreira J, Lamond AI: Nuclear organization of splicing snRNPs during differentiation of murine erythroleukemia cells in vitro. J Cell Biol 123: 1055-1068 (1993)

Bleichert F, Baserga SJ: The long unwinding road of RNA helicases. Mol Cell 27:339-352 (2007)

Bleoo S, Sun X, Hendzel MJ, Rowe JM, Packer M, Godbout R: Association of human DEAD box protein DDX1 with a cleavage stimulation factor involved in $3^{\prime}$-end processing of pre-mRNA. Mol Biol Cell 12:3046-3059 (2001).

Braun RE: Post-transcriptional control of gene expression during spermatogenesis. Semin Cell Dev Biol 9:483-489 (1998).

- Cartegni L, Krainer AR: Disruption of an SF2/ASFdependent exonic splicing enhancer in SMN2 causes spinal muscular atrophy in the absence of SMN1. Nat Genet 30:377-384 (2002).

-Chandler SD, Mayeda A, Yeakley JM, Krainer AR, Fu XD: RNA splicing specificity determined by the coordinated action of RNA recognition motifs in SR proteins. Proc Natl Acad Sci USA 94: 3596-3601 (1997)

Chusainow J, Ajuh PM, Trinkle-Mulcahy L, Sleeman JE, Ellenberg J, Lamond AI: FRET analyses of the U2AF complex localize the U2AF35/ U2AF65 interaction in vivo and reveal a novel self-interaction of U2AF35. RNA 11:1201-1214 (2005).

Dellaire G, Makarov EM, Cowger JJ, Longman D, Sutherland HG, et al: Mammalian PRP4 kinase copurifies and interacts with components of both the U5 snRNP and the N-CoR deacetylase complexes. Mol Cell Biol 22:5141-5156 (2002).

Du K, Taub R: Alternative splicing and structure of the human and mouse SFRS5/HRS/SRp40 genes. Gene 204:243-249 (1997).

-Elliott DJ, Oghene K, Makarov G, Makarova O, Hargreave TB, et al: Dynamic changes in the subnuclear organisation of pre-mRNA splicing proteins and RBM during human germ cell development. J Cell Sci 111:1255-1265 (1998).

Erickson RP: Post-meiotic gene expression. Trends Genet 6:264-269 (1990)

Foresta C, Ferlin A, Moro E: Deletion and expression analysis of $A Z F a$ genes on the human $Y$ chromosome revealed a major role for $D B Y$ in male infertility. Hum Mol Genet 9:1161-1169 (2000).

Godbout R, Squire J: Amplification of DEAD box protein gene in retinoblastoma cell lines. Proc Natl Acad Sci USA 90:7578-7582 (1993).
Guth S, Tange TO, Kellenberger E, Valcarcel J: Dual Ogawa H, Ueda T, Aoyama T, Aronheim A, Nagata function for $\mathrm{U} 2 \mathrm{AF}(35)$ in AG-dependent premRNA splicing. Mol Cell Biol 21:7673-7681 (2001).

Hofman MC, Narisawa S, Hess RA, Milan JL: Immortalization of germ cells and somatic testicular cells using the SV40 large T antigen. Exp Cell Res 201:417-435 (1992).

Jumaa H, Guenet JL, Nielsen PJ: Regulated expression and RNA processing of transcripts from the Srp20 splicing factor gene during the cell cycle. Mol Cell Biol 17:3116-324 (1997)

Leroy P, Alzari P, Sassoon D, Wolgemuth D, Fellous $\mathrm{M}$ : The protein encoded by a murine male germ cell-specific transcript is a putative ATP-dependent RNA helicase. Cell 57:549-559 (1989).

Luking A, Stahl U, Schmidt U: The protein family of RNA helicases. Crit Rev Biochem Mol Biol 33:259-296 (1998).

Lyon MF, Searle AG: Genetic variants and strains of the laboratory mouse, 2nd ed. (Oxford University Press, Oxford 1989).

Mayeda A, Screaton GR, Chandler SD, Fu XD, Krainer AR: Substrate specificities of SR proteins in constitutive splicing are determined by their RNA recognition motifs and composite pre-mRNA exonic elements. Mol Cell Biol 19: 1853-1863 (1999).

Meissner RM, Lopato S, Gotzmann J, Sauermann G, Barta A: Proto-oncoprotein TLS/FUS is associated to the nuclear matrix and complexed with splicing factors PTB, SRm160, and SR proteins. Exp Cell Res 15:184-195 (2003).

Merendino L, Guth S, Bilbao D, Martinez C, Valcarcel J: Inhibition of $m s l-2$ splicing by Sex-lethal reveals interaction between U2AF35 and the 3' splice site AG. Nature 402:838-841 (1999).

Mollet I, Barbosa-Morais NL, Andrade J, CarmoFonseca M: Diversity of human U2AF splicing factors. FEBS J 273:4807-4816 (2006).

Monesi V: Ribonucleic acid synthesis during mitosis and meiosis in the mouse testis. J Cell Biol 22:521-532 (1964).

Moussa F, Oko R, Hermo L: The immunolocalization of small nuclear ribonucleoprotein particles in testicular cells during the cycle of the seminiferous epithelium in the adult rat. Cell Tissue Res 278:363-378 (1994).

Neish AS, Anderson SF, Schlegel BP, Wie W, Parvin JD: Factors associated with the mammalian RNA polymerase II holoenzyme. Nucleic Acids Res 26:847-853 (1998). S, Fukunaga R: A SWI2/SNF2-type ATPase/helicase protein, mDomino, interacts with myeloid zinc finger protein 2A (MZF-2A) to regulate its transcriptional activity. Genes Cells 8: 325-339 (2003).

Oltra E, Werner R: Cloning of a potential regulatory protein involved in connexin 43 gene expression, in Werner R (ed): Gap junctions, pp 321-325 (IOS Press, Amsterdam 1998).

Oltra E, Pfeifer I, Werner I: Ini, a small nuclear protein that enhances the response of the connexin43 gene to estrogen. Endocrinology 144: 3148-3158 (2003).

Pacheco TR, Gomes AQ, Barbosa-Morais NL, Benes V, Ansorge W, et al: Diversity of vertebrate splicing factor U2AF35: identification of alternatively spliced U2AF1 mRNAS. J Biol Chem 279:27039-2749 (2004).

- Park E, Han J, Son GH, Lee MS, Chung S, et al: Cooperative actions of Tra2alpha with $9 \mathrm{G} 8$ and SRp30c in the RNA splicing of the gonadotropin-releasing hormone gene transcript. J Biol Chem 281:401-409 (2006).

Ruskin B, Zamore PD, Green MR: A factor, U2AF is required for $\mathrm{U} 2 \mathrm{snRNP}$ binding and splicing complex assembly. Cell 52:207-219 (1988).

Santama N, Dotti CG, Lamond AI: Neuronal differentiation in the rat hippocampus involves a stage-specific reorganization of subnuclear structure both in vivo and in vitro. Eur J Neurosci 8:892-905 (1996).

Silver LM: Mouse Genetics (Oxford University Press, Oxford 1995) http://www.informatics. jax.org/silver/.

Snow BE, Heng HH, Shi XM, Zhou Y, Du K, et al: Expression analysis and chromosomal assignment of the human SFRS5/SRp40 gene. Genomics 43:165-170 (1997).

Steger K: Haploid spermatids exhibit translationally repressed mRNAs. Anat Embryol (Berl) 203:323-334 (2001)

Tanner NK, Linder P: DExD/H box RNA helicases: from generic motors to specific dissociation functions. Mol Cell 8:251-262 (2001).

Tascou S, Nayernia K, Samani A, Schmidtke J, Vogel $\mathrm{T}$, et al: Immortalization of murine male germ cells at a discrete stage of differentiation by a novel directed promoter-based selection strategy. Biol Reprod 63:1555-1561 (2000).

Trappe R, Ahmed M, Glaser B, Vogel C, Tascou S, et al: Identification and characterization of a novel murine multigene family containing a PHD-finger-like motif. Biochem Biophys Res Commun 293:816-826 (2002a). 
Trappe R, Schulze E, Rzymski T, Frode S, Engel W: The Caenorhabditis elegans ortholog of human PHF5a shows a muscle-specific expression domain and is essential for C. elegans morphogenetic development. Biochem Biophys Res Commun 297:1049-1057 (2002b).

Ueda T, Watanabe-Fukunaga R, Ogawa H, Fukuyama $\mathrm{H}$, Higashi $\mathrm{Y}$, et al: Critical role of the $\mathrm{p} 400 / \mathrm{mDomino}$ chromatin-remodeling ATPase in embryonic hematopoiesis. Genes Cells 12:581-592 (2007).

Venables JP, Eperon I: The roles of RNA-binding proteins in spermatogenesis and male infertility. Curr Opin Genet Dev 9:346-354 (1999).

Wang Q, Rymond BC: Rds3p is required for stable U2 snRNP recruitment to the splicing apparatus. Mol Cell Biol 23:7339-7349 (2003).
Will CL, Urlaub H, Achsel T, Gentzel M, Wilm M, Luhrmann R: Characterization of novel SF3b and $17 \mathrm{~S}$ U2 snRNP proteins, including a human Prp5p homologue and an SF3b DEAD-box protein. EMBO J 21:4978-4988 (2002).

Wilson CJ, Chao DM, Imbalzano AN, Schnitzler GR, Kingston RE, Young RA: RNA polymerase II holoenzyme contains SWI/SNF regulators involved in chromatin remodelling. Cell 26: 235-244 (1996).

Wu JY, Maniatis T: Specific interactions between proteins implicated in splice site selection and regulated alternative splicing. Cell 17:10611070 (1993).

Wu S, Romfo CM, Nilsen TW, Green MR: Functional recognition of the 39 splice site AG by the splicing factor U2AF35. Nature 402:832-835 (1999).
Zamore PD, Patton JG, Green MR: Cloning and domain structure of the mammalian splicing factor U2AF. Nature 355:609-614 (1992).

Zhang M, Zamore PD, Carmo-Fonseca M, Lamond AI, Green MR: Cloning and intracellular localization of the U2 small nuclear ribonucleoprotein auxiliary factor small subunit. Proc Natl Acad Sci USA 89:8769-8773 (1992).

Zimmermann S, Steding G, Emmen JMA, Brinkmann AO, Nayernia K, et al: Targeted disruption of the Insl3 gene causes bilateral cryptorchidism. Mol Endocrinol 13:681-691 (1999).

Zuo P, Maniatis T: The splicing factor U2AF35 mediates critical protein-protein interactions in constitutive and enhancer-dependent splicing. Genes Dev 10:1356-1368 (1996). 